\title{
Formulation of a mapping formula to estimate well- being utility from the clinical subjective well-being scales
}

\author{
Akihiro Koreki \\ Keio University School of Medicine \\ Maki Nagaoka \\ Keio University School of Medicine \\ Akira Ninomiya \\ Keio University School of Medicine \\ Masaru Mimura \\ Keio University School of Medicine \\ Mitsuhiro Sado ( $\nabla$ mitsusado@keio.jp ) \\ Keio University School of Medicine
}

\section{Research Article}

Keywords: health economic evaluation, cost-effective analysis, mapping formula, ICECAP-A, SWLS, Flourishing Scale, SPANE

Posted Date: February 28th, 2022

DOI: https://doi.org/10.21203/rs.3.rs-1394690/v1

License: (c) (i) This work is licensed under a Creative Commons Attribution 4.0 International License. Read Full License 


\section{Abstract}

Background: Cost-effective analysis is one of the most useful analyses for political decision-making in medicine under a limited budget. Although the data of the Investigating Choice Experiments Capability Measure for Adults (ICECAP-A) is sometimes essential for the measurement of cost effectiveness, such data is often lacking in most clinical trials. Therefore, a conversion formula (i.e. mapping) derived from the values of clinical assessment scales into utility is required.

Methods: We used an internet survey where participants were asked to fill in four kinds of self-reported questionnaires (ICECAP-A, the Satisfaction with Life Scale (SWLS), Flourishing Scale (FS), and the Scale of Positive and Negative Experience (SPANE)). A beta regression was conducted with the utility assessed by ICECAP-A as a dependent variable.

Results: We developed several mapping formulae depending on available questionnaires. These mapping formulae were well-validated in our validated sample. The models using more number of questionnaires tended to show better mapping.

Conclusions: The mapping function of our formula was within the range of other reported mapping studies. We believe this formula is useful for cost effective analyses of several trials where utility data is lacking.

Clinical Trials Registry identifier: UMIN:R000051079.

\section{Background}

Health economic evaluations are conducted to make informed health care resource allocation decisions. ${ }^{1}$ This is significantly important for the optimal allocation of restricted resources. Cost effectiveness analysis is the gold standard of economic evaluation in the healthcare field. It measures consequences in natural units, such as life-years gained, disability days avoided, or cases detected. ${ }^{2}$ Cost effectiveness is assessed by the Incremental Cost Effectiveness Ratio (ICER), obtained by calculating the difference in costs between two health care programs divided by the difference in outcomes between the programs, with the comparison typically being between a new health care program and the existing approach to dealing with the same patient group. ${ }^{3}$ Quality Adjusted Life Years (QALYs) is the indicator typically used for clinical outcomes because it ensures comparability among different health conditions. The healthrelated utility score, which is measured by patient-reported outcomes, is required to calculate QALYS. EuroQol 5 Dimensions (EQ5D) is a scale which assesses the health conditions of respondents based on five dimensions: mobility, self-care, usual activities, pain/discomfort, anxiety/depression. ${ }^{4}$

However, several have raised the idea that measuring health alone is inadequate. ${ }^{5-7}$ This is especially the case when the well-being of the targets of an intervention is improving, rather than their physical/mental function, and where the effects of such interventions are anticipated to ripple outside the recipients to their carers, families, or the general public. In these cases, any scale requires a function to assess well- 
being from a broader perspective. The Investigating Choice Experiments Capability Measure for Adults (ICECAP-A) is a scale which is expected to address this issue. ${ }^{8}$ It is a scale which has introduced Sen's capability approach ${ }^{9,10}$; it assesses a person's ability to achieve important 'functioning'. It is a self-report scale which consists of five attributes with four levels ranging from 'full capability' to 'no capability'. The attributes involved in the scale are as follows: 1) Stability (being able to feel settled and secure), 2) Attachment (being able to have love, friendship, and support), 3) Autonomy (being able to be independent), 4) Achievement (being able to achieve and progress), 5) Enjoyment (being able to have enjoyment and pleasure). In this manner, attention has been drawn to well-being in the health economics field.

Like in the health economics field, well-being has attracted significant attention in the field of clinical trials, and relevant studies have been rapidly emerging. The common scales to assess subjective wellbeing are the Satisfaction with Life Scale (SWLS), Flourishing Scale (FS), and the Scale of Positive and Negative Experience (SPANE). However, as discussed above, most existing clinical trials tend to solely use clinical scales and omit using ICECAP-A data required for economic evaluation. This had led to the necessity of a mapping formula derived from these values of clinical assessment scales for effective economic evaluation. In the present study, we decided to develop a mapping formula to convert the scores of the well-being clinical scales to the utility scores weighted by ICECAP-A.

\section{Methods}

\section{Design}

A cross-sectional internet survey study was conducted.

\section{Participants}

The participants of the survey were general residents recruited through the survey system offered by Macromill (https://www.macromill.com/). The candidates were invited to the survey if they were general residents (no particular restrictions on health conditions, such as past illnesses), in the age group of 20 to 65 years, and were able to understand the purpose of the survey and accordingly provide their consent. There were no particular exclusion criteria.

\section{Procedures}

We conducted the internet-survey using the system provided by Macromill. They sent an email to the registered samples to announce this study. The candidates who showed interest and were ascertained as eligible for the study were offered a written leaflet which provided detailed information about the research. Once they provided their consent through the survey system, they were requested to fill four selfreported questionnaires related to well-being. 


\section{Clinical scales}

The scales included in the survey were ICECAP-A, SWLS, FS, and SPANE. It is to be noted that the notion of well-being is complex and there is no consensus among researchers regarding its parameters. However, to date, it is thought that well-being consists of different aspects and measuring such aspects is crucial in providing comprehensive snapshots of the respondents' well-being (OECD, 2013). ${ }^{11}$ The Organisation for Economic Co-operation and Development (OECD) has prescribed guidelines on measuring subjective well-being. Therefore, in this survey, we requested participants to fill the following three clinical scales, in addition to ICECAP-A, each of which measures a different aspect of well-being (SWLS: cognitive; SPANE: affect; FS: eudemonic aspect of well-being).

ICECAP-A: ICECAP-A was developed to measure the capability well-being of adults, which was not captured adequately in pre-existing health-related quality of life scales. It consists of 5 attributes: attachment (ability to have love, friendship, and support), stability (ability to feel settled and secure), achievement (ability to achieve and progress in life), enjoyment (ability to experience enjoyment and pleasure), and autonomy (ability to be independent), each of which has four levels. It provides a single index value for well-being utility ranging between 0 and 1 . A higher score indicates a better well-being status. $^{8}$

\section{SWLS}

This scale is a 5-item self-reported questionnaire to evaluate the cognitive aspect of subjective well-being. Scores for each subscale range from $1=$ strongly disagree to $7=$ strongly agree. Total scores range from 5 to 35 , with higher scores indicating higher satisfaction. ${ }^{12}$

\section{FS}

This scale includes eight items relevant to significant aspects of human functioning, ranging from positive relationships to feelings of competence, meaning, and having a purpose in life. FS is recognized as a scale which measures the eudemonic aspect of subjective well-being. Each item is answered on a 1-7 scale that ranges from strong disagreement to strong agreement. Possible scores range between 8 and 56. A higher score indicates that the respondent has a better eudemonic well-being. ${ }^{13}$

\section{SPANE}

SPANE consists of twelve items, including six items to assess positive experiences and six items to assess negative experiences. ${ }^{13}$ The positive score (SPANE-P) and the negative score (SPANE-N) are in the range of 6 to 30. A higher score means higher positive or negative affective aspects of well-being. The 
two scores can be combined by subtracting the negative score from the positive one (SPANE-B, score range: -24-24).

\section{Statistics}

In statistics, to predict the score of ICECAP-A, a beta regression was conducted, with utility assessed by ICECAP-A as a dependent variable, because this score ranges from 0 to 1 , which is beta distribution. Since the tariff of ICECAP-A among Japan's population is not still available, we used the weighted utility scores by ICECAP-A among UK samples. Age, sex, SWLS, FS, and SPANE were set as independent variables. Several statistical models were developed (i.e. A-G: total score was used, a-g: each sub-score was used) (Table 1), and fitting levels were compared using the mean absolute error (MAE) and the root mean squared error (RMSE) because those error values provide better means to assess mapping functions than R-squared, which focuses on how well the model explains the dataset it was estimated on. ${ }^{14}$ The independent variables in each model were as below:

$<$ Independent variables in each model $>$

Model name:

-Uppercase means total score was used, while lowercase means each subscale was used

-Number means the number of questionnaires used in the model

- model 1A/a: SWLS, model 1B/b: FS, model 1C/c: SPANE-P, model 1D/d: SPANE-N

- model 2A/a: SWLS and FS, model 2B/b: SWLS and SPANE-P, model 2C/c: SWLS and SPANE-N, model 2D/d: FS and SPANE-P, model 2E/e: FS and SPANE-N, model 2F/f: SPANE-P and SPANE-N

- model 3A/a: SWLS, FS and SPANE-P, model 3B/b: SWLS, FS and SPANE-N, model 3C/c: SWLS, SPANE-P and SPANE-N, model 3D/d: FS, SPANE-P and SPANE-N

- model 4A/a: SWLS, FS, SPANE-P and SPANE-N

All the participants were divided into two groups. Three hundred participants were randomly selected, and their data were used for the development of the model (estimation sample). The data of the remaining participants were used to assess the validity of the developed model (validation sample). These analyses were conducted using R (4.1.0).

\section{Results}

Fifty hundred fifteen individuals (age: $44.1 \pm 11.6$ years old, 297 females, utility assessed by ICECAP-A: $0.62 \pm 0.21$, SWLS: $19.7 \pm 7.1$, FS: $32.8 \pm 9.3$, SPANE-P/N: $19.2 \pm 5.3 / 18.3 \pm 4.7$ ) answered these questionnaires using the internet survey. The data in the estimation sample and validation sample were like that of total sample, and there was no significant difference between them (Table 1). ICECAP-A was 
correlated with SWLS, FS, SPANE-P, and SPANE-N $(r=0.65,0.67,0.65$ and -0.47 , respectively), SWLS was correlated with FS, SPANE-P, and SPANE-N ( $r=0.76,0.67$ and -0.43 , respectively), FS was correlated with SPANE-P and SPANE-N ( $r=0.74$ and -0.39 , respectively). All $p$-values of correlation analyses were $<0.01$.

The modelling and fitting in each model are shown in Table 2. In total, the model using more numbers of questionnaires showed better modelling and fitting. The model using subscales did not show any significant changes; rather, its results worsened. The smallest RMSE and MAE were found in Model 4A, where all the questionnaires were used. The beta of our recommended models is shown in Table 3.

\section{Discussion}

We developed a mapping formula using subjective well-being questionnaires (SWLS, FS, and SPANE) for the utility assessed by ICECAP-A. To the best of our knowledge, this is the first mapping formula to assess utility scores weighted by ICECAP-A from the aforementioned subjective well-being scales. These models were well-validated in our validated sample. The models using questionnaires tended to have better fitting. Given that which mapping formula is required is based on which questionnaire is included in the original research, we have developed several models, including models using only one questionnaire.

In our model, RMSE and MAE, which are used for assessing mapping functions and where smaller values of errors means better mapping function, the RMSE was $0.14-0.16$, and the MAE was $0.10-0.12$, which are within the range of other reported mapping studies (MSE: 0.084-0.2 and MAE: 0.0011 to 0.19). ${ }^{14}$ Models using more questionnaires showed smaller RMSEs and MAEs. Given that SWLS, FS, and SPANE can assess slightly different aspects of well-being, this finding is convincing. In contrast, models using subscales did not show any great improvement and showed slightly higher RMSEs and MAEs than models using the total score.

In addition to predictive validity, where errors were assessed, the contextual similarity among the questionnaires should be discussed as content validity. Given the focus of ICECAP-A on attachment, stability, achievement, enjoyment, and autonomy, ${ }^{8}$ in our estimation, the ICECAP-A score could be related more to FS (psychological well-being), than SWLS (cognitive well-being) and SPANE (affective wellbeing). ${ }^{12,13}$ This is because the concept of psychological well-being is developed based on eudaemonia, which includes the sense of achievement and the sense of sufficiency, while the concept of affective wellbeing is developed based on a hedonic perspective. ${ }^{12,13,15}$ In truth, FS had the highest correlation with ICECAP-A, although such difference was not huge compared to the correlations of other two questionnaires (i.e. SWLS and SPANE-P) with ICECAP. On the other hand, the improvement of the mapping function upon using more questionnaires suggested that slight differences in the aspects of well-being existed among the questionnaires. In the OCED model, life satisfaction, positive affect and eudaemonic well-being are listed as an equal level measurement concept. ${ }^{11}$ 
Our present study has several limitations. First, it was difficult to predict lower values of ICECAP-A because the minimum mapped value was 0.10 , while the actual minimum value was 0.00 . Second, our mapping function was reasonable but not perfect, indicating that there were other factors which affect the ICECAP-A score other than the three questionnaires used in this study. However, given that data is usually limited when mapping is required, our formula is reasonable from a practical perspective. Finally, as discussed previously, the utility weighted by ICECAP-A in the study was derived from UK samples, due to the unavailability of the tariff among Japanese samples. Given that the value of well-being should also be in line with the given culture of samples, one should be aware of such limitation when applying this formula. In this vein, the development of the ICECAP-A tariff among Japanese samples and the mapping formula based on it can be expected in the near future.

In conclusion, we have developed several mapping formulae from questionnaires (SWLS, FS, and SPANE) to derive the utility assessed by ICECAP-A. These formulas were validated in our sample. Researchers may choose the appropriate mapping formula depending on which questionnaire is included in their original research.

\section{Declarations}

\section{Ethics approval and consent to participate}

This study was approved by the ethics review committee of Keio University School of Medicine (20210096), and is registered under the UMIN Clinical Trials Registry identifier: UMIN:R000051079. The candidates provided their consent on the website.

\section{Consent for publication}

Not applicable

\section{Availability of data}

The datasets generated during the current study are available from the corresponding author (MS) upon reasonable request.

\section{Competing interests}

The authors report no conflicts of interest regarding this study.

\section{Funding}


This work was supported by Edoga Inc. The funding source had no role in the study design, data collection, management, analysis, interpretation of the data, or in the writing of the paper.

\section{Authors' contributions}

AK and MS designed the study and drafted the study protocol. MN, AN, and MM refined the study protocol and provided technical suggestions. All authors read and approved the final manuscript

\section{Acknowledgements}

We would like to thank all patients who answered our questionnaire and also the staff for supporting this study.

\section{References}

1. Husereau D, Drummond M, Petrou S, et al. ISPOR Health Economic Evaluation Publication Guidelines-CHEERS Good Reporting Practices Task Force. Consolidated Health Economic Evaluation Reporting Standards (CHEERS)-explanation and elaboration: a report of the ISPOR Health Economic Evaluation Publication Guidelines Good Reporting Practices Task Force. Value Health. 2013 MarApr;16(2):231-50. doi: 10.1016/j.jval.2013.02.002.

2. Drummond MF, Sculpher MJ, Torrance G, et al. Methods for the Economic Evaluation of Health Care Programmes (3rd ed), Oxford University Press, New York (2005).

3. Gafni A, Birch S. Incremental cost-effectiveness ratios (ICERs): the silence of the lambda. Soc Sci Med. 2006 May;62(9):2091-100. doi:10.1016/j.socscimed.2005.10.023.

4. EuroQol G. EuroQol-a new facility for the measurement of health-related quality of life. Health Policy 1990; 16: 199-208.

5. Oliver A, Healey A, Donaldson C. Choosing the method to match the perspective: economic assessment and its implications for health-services efficiency. Lancet. 2002 May 18;359(9319):17714. doi: 10.1016/S0140-6736(02)08664-6.

6. Coast J. Is economic evaluation in touch with society's health values? BMJ. $2004 \mathrm{Nov}$ 20;329(7476):1233-6. doi: 10.1207/s15327752jpa4901_13.

7. Ryan M, Netten A, Skåtun D, Smith P. Using discrete choice experiments to estimate a preferencebased measure of outcome--an application to social care for older people. J Health Econ. 2006 Sep;25(5):927-44. doi: 10.1016/j.jhealeco.2006.01.001.

8. Al-Janabi H, Flynn T, Coast J. (2012) Development of a self-report measure of capability wellbeing for adults: the ICECAP-A. Quality of Life Research. 2012;21: 167-176. Doi: 10.1007/s11136-011-99272.

9. Sen A. Inequality reexamined. New York: Russell Sage Foundation; 1992. 
10. Sen A. Capability and well-being. In: Nussbaum MC, editor. The quality of life. Oxford: Clarendon Press; 1993.

11. OECD (2013). OECD guidelines on measuring subjective well-being. Paris: OECD Publishing.

12. Diener E, Emmons RA, Larsen RJ, Griffin S. The Satisfaction With Life Scale. J Pers Assess. 1985 Feb;49(1):71-5. doi: 10.1207/s15327752jpa4901_13.

13. Diener, E., Tov, W., Kim-Prieto, C., Choi, D., Oishi, S., and Biswas Diener, R. (2010). New well-being measures: short scales to assess flourishing and positive and negative feelings. Soc. Indic. Res. 97, 143-156. doi: 10.1007/s11205-009-9493-y.

14. Brazier JE, Yang Y, Tsuchiya A, Rowen DL. A review of studies mapping (or cross walking) nonpreference based measures of health to generic preference-based measures. Eur J Health Econ. 2010 Apr;11(2):215-25. doi: 10.1007/s10198-009-0168-z.

15. Sirgy MJ. Positive balance: a hierarchical perspective of positive mental health. Qual Life Res. 2019 Jul;28(7):1921-1930. doi: 10.1007/s11136-019-02145-5.

\section{Tables}

Tables are only available as a download in the Supplemental Files section.

\section{Figures}


$\begin{array}{llllll}0.0 & 0.2 & 0.4 & 0.6 & 0.8 & 1,0\end{array}$
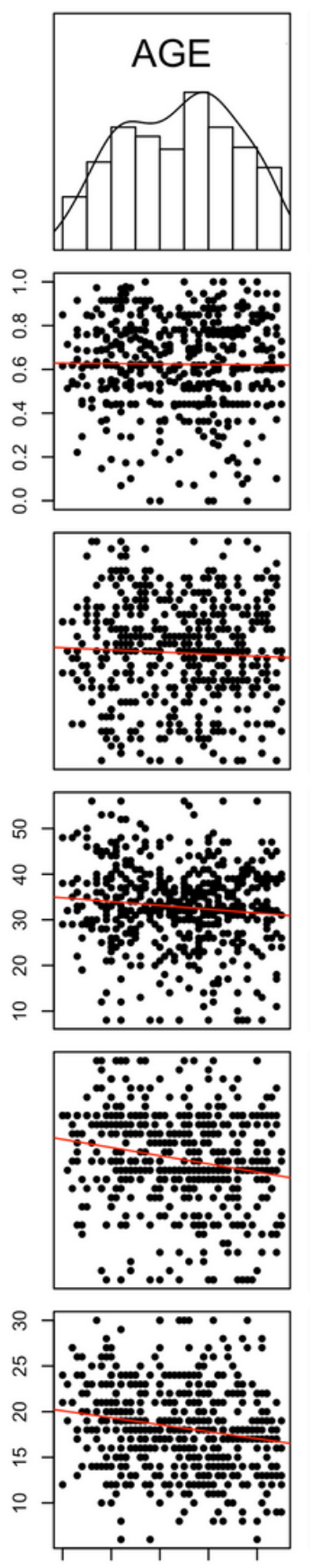

$\begin{array}{lllll}20 & 30 & 40 & 50 & 60\end{array}$

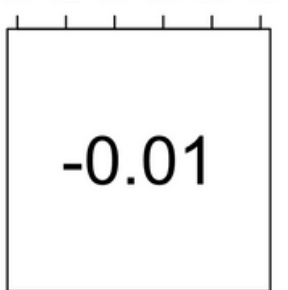

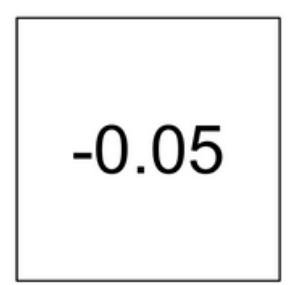
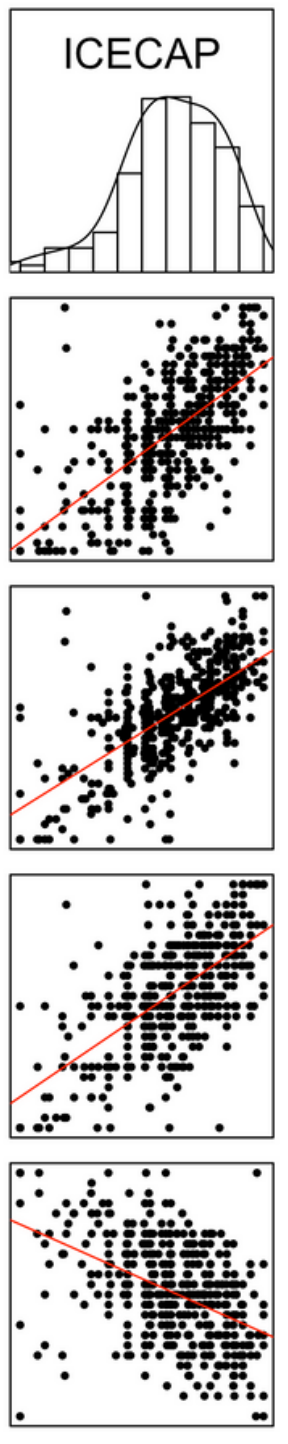
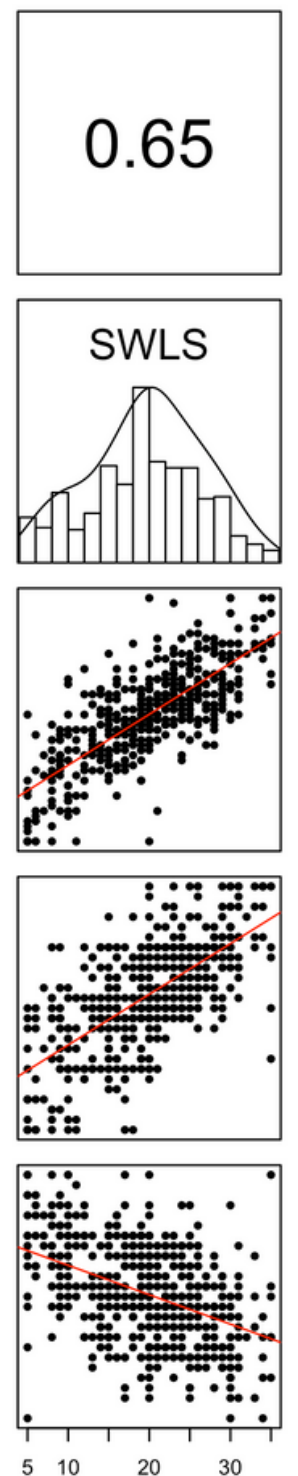

$\begin{array}{lllll}10 & 20 & 30 & 40 & 50\end{array}$
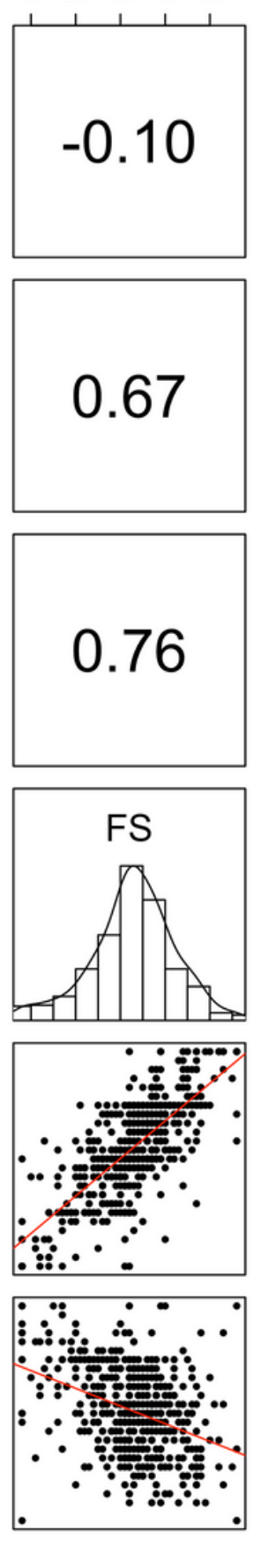

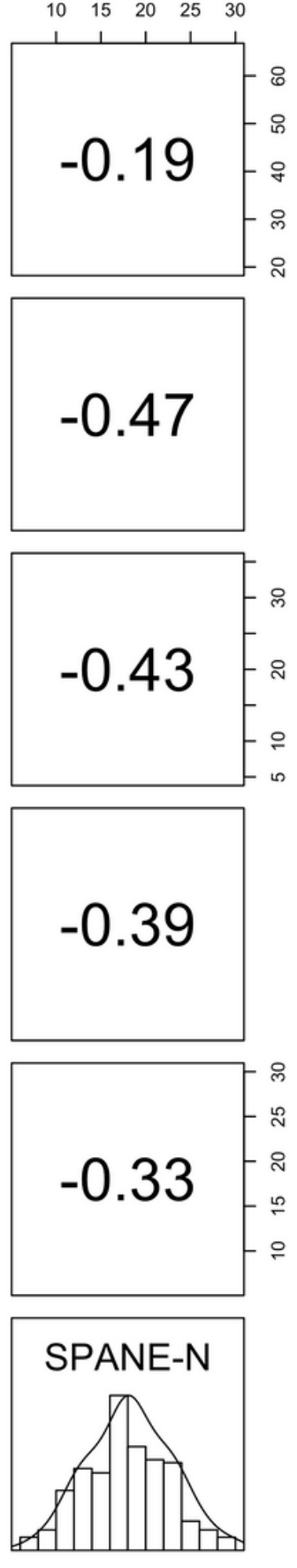

Figure 1

Legend not included with this version

\section{Supplementary Files}

This is a list of supplementary files associated with this preprint. Click to download. 
- TableMapping.xlsx

Page $11 / 11$ 